\title{
Fatores associados à prática de caminhada, atividades físicas moderadas e vigorosas em adolescentes
}

\author{
Factors associated with walking, moderate and vigorous physical \\ activities in adolescents
}

\author{
V.C. Barbosa Filho, A.P. Gordia, T.M. Bianchini de Quadros, E.A. Souza, W. Campos
}

\begin{abstract}
Este estudo objetivou investigar a associação do sexo, faixa etária, classe econômica, escolaridade do pai e estado nutricional com a prática de caminhada e atividades físicas moderadas e vigorosas em adolescentes. Participaram do estudo 578 escolares (305 meninos), com idades entre 11 e 16 anos, de uma escola pública do município de Fortaleza, Ceará, Brasil. As informações sobre a prática de atividades físicas foram obtidas através do International Physical Activity Questionnaire (IPAQ, versão 8, forma curta). A análise dos dados apropriou-se da estatística descritiva, Qui-quadrado, teste $t$ independente e análise de variância one-way. Treze por cento dos adolescentes foram classificados como inativos, sem diferença significativa entre os sexos $(p>.05)$. As moças apresentaram mais tempo despendido na prática de caminhada $(p=.019)$ e em atividades vigorosas $(p=.017) \mathrm{em}$ comparação aos rapazes. Adolescentes da faixa etária de 14-16 anos tiveram maior participação em caminhada em comparação aos de 11-12 anos $(p=.026)$ e 13 anos $(p=.006)$. Verificou-se associação positiva entre a classe econômica e o tempo gasto em atividades físicas moderadas $(p=.034)$. Em conclusão, a associação do sexo, faixa etária e classe econômica com a prática de atividade física em adolescentes varia de acordo com o tipo e intensidade de atividade realizada.

Palavras-chave: atividade física, comportamento do adolescente, fatores socioeconômicos, excesso de peso, adolescentes
\end{abstract}

ABSTRACT

This study aimed at investigating the relationship between a set of variables (adolescents' gender, age, economic level, nutritional status, and fathers' educational level) and physical activity practices. The sample consisted of 578 students from a public school, aged between 11 and 16 years. The International Physical Activity Questionnaire (IPAQ, version 8, short form) was applied in order to measure their physical activity practices. Chi-square, independent $t$ test and one-way ANOVA were implemented. Thirteen percent of adolescents were physically inactive and no significant difference was found between gender $(p>.05)$. Compared to boys, girls spent more time in walking $(p=.019)$ and vigorous activities $(p=.017)$. Adolescents aged 14-16 years spent more time in walking compared with those aged 11-12 years $(p=.026)$ and 13-year-olds $(p=.006)$. There was a positive association between economic level and time spent in moderate physical activity $(p=.034)$.

Keywords: physical activity, adolescent behavior, socioeconomic factors, overweight, adolescents

Submetido: 25.07.2010 | Aceite: 24.10.2010

Valter Cordeiro Barbosa Filho. Grupo de Pesquisa em Promoção e Diagnóstico da Saúde na Adolescência, Universidade Federal do Ceará, Fortaleza, Brasil; Universidade Federal do Paraná, Curitiba, Brasil. Bolsista CAPES.

Alex Pinheiro Gordia e Teresa Maria Bianchini de Quadros. Universidade Federal do Recôncavo da Bahia, Amargosa, BA, Brasil.

Evanice Avelino de Souza. Universidade de Brasília, Brasília, DF, Brasil.

Wagner de Campos. Universidade Federal do Paraná, Curitiba, PR, Brasil.

Endereço para correspondência: Valter Cordeiro Barbosa Filho. Rua Sanito Rocha, n.135, ap.504, Bairro Cristo

Rei, CEP: 80050-380 - Curitiba, Paraná, Brasil.

E-mail: valtercbf@gmail.com 
A atividade física pode ser definida como qualquer movimento corporal produzido pelo sistema músculo-esquelético que resulta em gasto energético acima do repouso (Caspersen, Powell, \& Christenson, 1985). Em crianças e adolescentes, estas atividades são comumente realizadas ao caminhar até a escola, participar de atividades desportivas, brincar ativamente com amigos e realizar tarefas domésticas ou trabalhos manuais (Katzmarzyk et al., 2008).

A literatura preconiza que a prática regular de atividade física em indivíduos jovens está relacionada com a redução do risco de diversas doenças como a hipertensão, diabetes tipo 2 , aterosclerose e alguns tipos de câncer, e com a melhoria na capacidade dos sistemas músculoesquelético e cardiorrespiratório, além de favorecer o crescimento físico saudável e o controle de peso corporal (Strong et al., 2005). Há evidências de que estes benefícios podem ser observados ainda na juventude e são determinantes do estado de saúde na fase adulta (Boreham et al., 2002; Kvaavik, Klepp, Tell, Meyer, \& Batty, 2009; Twisk, Kemper, Van Mechelen, \& Post, 2001).

Entretanto, o estilo de vida moderno da população jovem é caracterizado pela menor quantidade de movimentos corporais no cotidiano, favorecendo o desenvolvimento de doenças crônicas não-transmissíveis (Marani, Oliveira, \& Guedes, 2006; Murphy, McNeilly, \& Murtagh, 2010). Desta forma, estudos têm investigado variáveis associadas à prática de atividade física em indivíduos jovens visando identificar os principais fatores que influenciam no estilo de vida ativo (Bastos, Araújo, \& Hallal, 2008; Gonçalves, Hallal, Amorim, Araújo, \& Menezes, 2007; GordonLarsen, McMurray, \& Popkin, 2000; Hallal, Bertoldi, Gonçalves, \& Victora, 2006).

Estudos prévios demonstram que a faixa etária, o sexo e a condição socioeconômica são alguns dos fatores comumente associados com a prática de atividades físicas em adolescentes (Ceschini, Andrade, Oliveira, Araújo Júnior, \& Matsudo, 2009; Farias Júnior, 2008; Nhantumbo, Maia, Saranga, \& Prista, 2008;
Seabra, Mendonça, Thomis, Anjos, \& Maia, 2008; Vasques \& Lopes, 2009). Contudo, são escassas as informações sobre a relação destes fatores com os diferentes tipos e intensidades de atividades físicas na adolescência (prática de caminhada, atividades físicas moderadas e vigorosas). Esta análise pode ser importante para indicar se existem fatores específicos associados com determinados tipos e intensidades de atividades físicas, bem como para identificar os grupos de maior risco ao estilo de vida inativo.

Portanto, o presente estudo objetivou analisar a associação do sexo, faixa etária, classe econômica, escolaridade do pai e estado nutricional com a prática de caminhada e atividades físicas moderadas e vigorosas em adolescentes, com idades entre 11 e 16 anos, de uma escola pública do município de Fortaleza, Ceará, Brasil.

\section{Amostra}

\section{MÉTODO}

O presente estudo foi realizado com adolescentes de uma escola pública do município de Fortaleza, Ceará, na região Nordeste do Brasil. Os pais e/ou responsáveis autorizaram a participação dos escolares na pesquisa mediante a assinatura do termo de consentimento livre e esclarecido (TCLE). Os procedimentos adotados no presente estudo foram aprovados pelo Comitê de Ética em Pesquisa da Universidade Federal do Ceará (protocolo $\mathrm{n}^{\circ}$ 244/08).

A amostra foi composta por 767 alunos regularmente matriculados na escola avaliada, distribuídos em 18 turmas do sétimo ao nono ano do ensino fundamental (ano letivo de 2009), todas no turno matutino. Destes, 189 adolescentes (24,6\% dos alunos matriculados) não participaram do presente estudo por: i) faltar o dia de entrega do TCLE; ii) não retornar o TCLE assinado pelo responsável; ou iii) preencher incorretamente os questionários. Desta forma, a amostra foi composta por 578 escolares ( $75,4 \%$ dos alunos matriculados), de ambos os sexos, com idades entre 11 e 16 
Tabela 1

Descrição da amostra em relação às variáveis idade, estatura, massa corporal e índice de massa corporal (IMC), com valores expressos em média $(M)$ e desvio padrão $(D P)$

\begin{tabular}{lccc}
\hline \multicolumn{1}{c}{ Variáveis } & Masculino $(n=305)$ & Feminino $(n=273)$ & Total $(n=578)$ \\
& $M \pm D P$ & $M \pm D P$ & $M \pm D P$ \\
\hline Idade $($ anos $)$ & $13.01 \pm 1.20$ & $12.86 \pm 1.10$ & $12.94 \pm 1.10$ \\
Estatura $(\mathrm{cm})$ & $159.00 \pm 10.10^{*}$ & $155.10 \pm 6.90^{*}$ & $157.20 \pm 8.90$ \\
Massa corporal $(\mathrm{kg})$ & $50.10 \pm 11.80$ & $48.50 \pm 9.90$ & $49.40 \pm 10.90$ \\
$\mathrm{IMC}\left(\mathrm{kg} / \mathrm{m}^{2}\right)$ & $19.64 \pm 3.30$ & $20.08 \pm 3.50$ & $19.85 \pm 3.40$ \\
${ }^{*} p<.01$ & &
\end{tabular}

anos. As características gerais da amostra de acordo com o sexo estão apresentadas na Tabela 1.

\section{Instrumentos e Procedimentos}

Previamente à coleta de dados, houve treinamento dos avaliadores com explicações teóricas e simulações práticas, visando à padronização tanto da aplicação de questionários quanto da avaliação antropométrica. A coleta de dados foi realizada em dois locais distintos: i) uma sala de aula para a aplicação dos questionários referentes à condição econômica e a atividade física; e ii) uma sala de aula para a avaliação antropométrica. Inicialmente, foram definidas turmas com até trinta adolescentes para o preenchimento dos questionários. Os adolescentes receberam as instruções referentes ao preenchimento dos questionários, sem ser estimado tempo para conclusão dos mesmos. Quando solicitado o auxílio dos avaliadores os escolares foram prontamente atendidos. Em seguida, os adolescentes foram encaminhados para a sala de avaliação antropométrica, para mensuração da estatura (cm) e da massa corporal $(\mathrm{kg})$. Todas as coletas foram realizadas no mês de março de 2009.

A mensuração da estatura e da massa corporal dos adolescentes foi realizada conforme padronização proposta por Gordon, Chumlea e Roche (1988). Para tanto, foi utilizada uma fita métrica (com escalas de .1 $\mathrm{cm}$, marca Easyread e modelo Cateb) fixada em uma parede plana, sem rodapé, para determinação da estatura; e uma balança digital (resolução de 100 g e capacidade de 150 $\mathrm{kg}$, marca Plenna e modelo Wind) para determinação da massa corporal. As medidas da massa corporal e estatura foram utilizadas para cálculo do Índice de Massa Corporal (IMC, $\mathrm{kg} / \mathrm{m}^{2}$ ). Para determinação do estado nutricional utilizou-se o critério brasileiro proposto por Conde e Monteiro (2006), classificando os adolescentes com excesso de peso (sobrepeso e obesidade) e peso normal.

Para avaliação da condição econômica foi utilizado o questionário da Associação Brasileira de Empresas de Pesquisa (ABEP, 2008) que visa estimar o poder de compra das pessoas e famílias e o grau de instrução do chefe da família (pai), separando nas classes econômicas A, B, C, D e E. As classes econômicas foram agrupadas em classe alta $(A+B)$, classe média $(C)$ e classe baixa $(D+E)$. Além disso, a escolaridade do chefe da família dos adolescentes foi considerada segundo o número de anos completos de escolaridade, sendo adotadas as seguintes categorias: $\leq 4$ anos, 5-8 anos, 9-11 anos e $\geq 12$ anos.

O nível de atividade física foi avaliado através do International Physical Activity Questionnaire (IPAQ, versão 8, forma curta). Essa versão é composta por oito questões abertas e suas informações permitem estimar o tempo despendido na última semana em caminhadas e atividades físicas de intensidade moderada ou vigorosa, além de estimar o tempo de inatividade física (posição sentada) 
(Matsudo et al., 2001). Este questionário foi validado para adolescentes brasileiros por Guedes, Lopes e Guedes (2005). O critério desenvolvido pelo IPAQ Research Committee (2005) foi utilizado para análise da atividade física dos adolescentes. Esta classificação considera os critérios de frequência e duração, e classifica o nível de atividade física dos adolescentes em três categorias: inativo; moderadamente ativo e muito ativo. Os adolescentes classificados nas duas últimas categorias foram considerados como ativos.

\section{Análise Estatística}

A análise dos dados apropriou-se da estatística descritiva, através de indicadores de média, desvio padrão e percentagem.

No que se refere à estatística inferencial, o teste do Qui-quadrado foi utilizado para verificar diferenças entre os sexos na proporção de adolescentes inativos em cada faixa etária. $\mathrm{O}$ teste $\mathrm{t}$ para amostra independente foi utilizado para verificar diferenças entre os sexos nas variáveis antropométricas, e entre os sexos e as classificações do estado nutricional no tempo semanal de atividade física. Para as variáveis com mais de três categorias (faixa etária, escolaridade do pai e classe econômica) utilizou-se a análise de variância one-way com post-hoc de Tukey para se verificar as diferenças entre os grupos no tempo semanal de atividade física.

Todos os resultados foram analisados através do SPSS 15.0, sendo adotado $p<.05$ como valor de significância estatística.

\section{RESULTADOS}

No presente estudo houve maior participação de adolescentes do sexo masculino (52.8\%) e da faixa etária de 11-12 anos (36.9\%). Foram verificadas médias semelhantes entre os sexos para a massa corporal e para o IMC, entretanto, os adolescentes do sexo masculino apresentaram média superior para a variável estatura do que seus pares do sexo feminino $(p<.001)$ (ver Tabela 1$)$.

A proporção de adolescentes classificados como inativos, de acordo com o sexo e faixa etária, pode ser observada na Figura 1. Treze por cento dos adolescentes foram classificados como inativos, sendo $15.1 \%$ do sexo masculino e $10.6 \%$ do feminino. Em relação ao nível de atividade física, não houve diferenças significativas entre os sexos na proporção de adolescentes inativos nas três faixas etárias e na amostra total $(p>.05)$.

A média semanal de participação em caminhadas, atividades físicas moderadas e

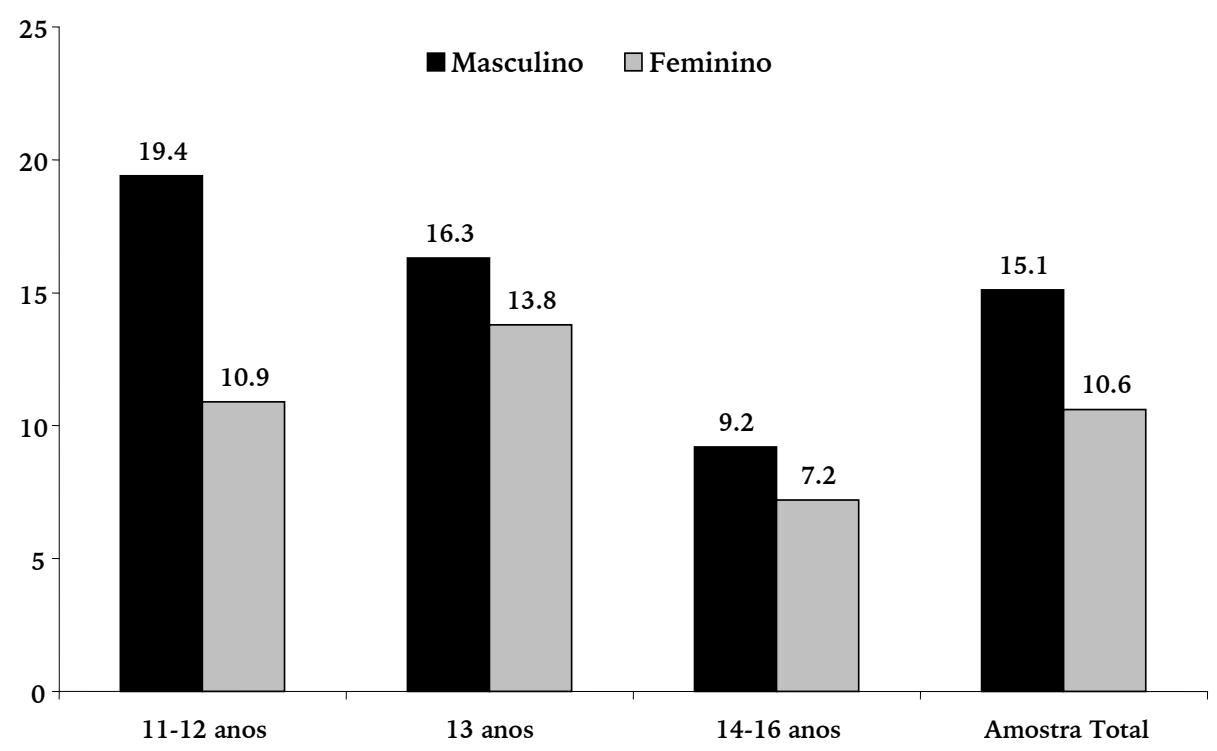

Figura 1. Proporção de adolescentes classificados como inativos de acordo com o sexo e faixa etária 
vigorosas de acordo com sexo, faixa etária, estado nutricional, escolaridade do pai e classe econômica pode ser observada na Tabela 2 . Verificou-se que as adolescentes do sexo feminino apresentaram mais tempo despendido com a prática de caminhada $(p=$ .019) e de atividades vigorosas $(p=.017)$ do que seus pares do sexo masculino. Moças também apresentaram média semanal de prática de atividades moderadas superior aos rapazes, embora a diferença não tenha sido estatisticamente significativa $(p=.055)$. Em relação às faixas etárias, observou-se maior participação de adolescentes da faixa etária de 14-16 anos em caminhada do que os adolescentes de 11-12 anos $(p=.026)$ e 13 anos de idade $(p=.006)$. Quanto à condição econômica, foi verificada diferença significativa $(p=.034)$ no tempo de atividade física moderada, sendo que os adolescentes de classe econômica mais elevada apresentaram média superior nestas atividades. Não foram verificadas diferenças significativas entre os grupos no tempo despendido em caminhada, atividades físicas moderadas ou vigorosas em relação ao estado nutricional e à escolaridade do pai $(p>.05)$.

\section{DISCUSSÃO}

A identificação dos fatores associados à prática de atividades físicas na adolescência é importante para orientar políticas públicas de

Tabela 2

Prática semanal de caminhada e atividades físicas moderadas e vigorosas em relação ao sexo, faixa etária, estado nutricional, escolaridade do pai e classe econômica, com valores expressos em média (M) e desvio padrão (DP)

\begin{tabular}{|c|c|c|c|c|}
\hline Variáveis & $\begin{array}{c}n(\%) \\
M \pm D P\end{array}$ & $\begin{array}{c}\text { Caminhada } \\
\text { (min/sem) } \\
M \pm D P\end{array}$ & $\begin{array}{c}\text { Moderada } \\
\text { (min/sem) } \\
M \pm D P\end{array}$ & $\begin{array}{c}\text { Vigorosa } \\
\text { (min/sem) } \\
M \pm D P\end{array}$ \\
\hline \multicolumn{5}{|l|}{ Sexo } \\
\hline Masculino & $305(52.8)$ & $312.9 \pm 455.5^{*}$ & $387.7 \pm 501.0$ & $217.9 \pm 321.2^{*}$ \\
\hline Feminino & $273(47.2)$ & $417.1 \pm 588.3^{*}$ & $498.0 \pm 818.2$ & $303.6 \pm 506.4^{*}$ \\
\hline \multicolumn{5}{|l|}{ Faixa etária } \\
\hline 11-12 anos & $213(36.9)$ & $329.5 \pm 550.6^{*}$ & $442.7 \pm 662.3$ & $234.3 \pm 442.0$ \\
\hline 13 anos & $184(31.8)$ & $297.4 \pm 447.3^{*}$ & $446.7 \pm 706.2$ & $273.6 \pm 452.2$ \\
\hline 14-16 anos & $181(31.3)$ & $466.2 \pm 552.6^{*}$ & $429.3 \pm 649.2$ & $271.3 \pm 358.6$ \\
\hline \multicolumn{5}{|l|}{ Estado nutricional } \\
\hline Peso normal & $404(69.9)$ & $357.0 \pm 489.5$ & $431.5 \pm 668.2$ & $243.7 \pm 414.8$ \\
\hline Excesso de peso & $174(30.1)$ & $373.9 \pm 599.7$ & $459.0 \pm 680.6$ & $292.5 \pm 433.8$ \\
\hline \multicolumn{5}{|l|}{ Escolaridade do Pai } \\
\hline$\leq 4$ anos & $237(41.0)$ & $389.9 \pm 556.5$ & $441.4 \pm 640.7$ & $276.3 \pm 429.3$ \\
\hline $5-8$ anos & $149(25.8)$ & $300.8 \pm 380.2$ & $350.7 \pm 494.4$ & $215.1 \pm 471.7$ \\
\hline 9-11 anos & $72(12.5)$ & $379.2 \pm 553.8$ & $548.4 \pm 984.2$ & $238.7 \pm 364.8$ \\
\hline$\geq 12$ anos & $120(20.8)$ & $372.9 \pm 524.6$ & $482.1 \pm 686.4$ & $288.6 \pm 420.8$ \\
\hline \multicolumn{5}{|l|}{ Classe econômica } \\
\hline Baixa $(D+E)$ & $342(59.2)$ & $371.1 \pm 555.7$ & $385.2 \pm 578.3^{*}$ & $252.0 \pm 462.4$ \\
\hline Média (C) & $197(34.1)$ & $348.0 \pm 477.0$ & $497.6 \pm 616.8^{*}$ & $259.5 \pm 352.9$ \\
\hline Alta $(A+B)$ & $39(6.7)$ & $354.3 \pm 482.2$ & $626.8 \pm 1342.9^{*}$ & $308.6 \pm 353.3$ \\
\hline Total & $578(100.0)$ & $362.1 \pm 524.6$ & $439.8 \pm 671.5$ & $258.4 \pm 420.8$ \\
\hline
\end{tabular}


promoção de atividade física (Sallis, Prochaska, \& Taylor, 2000). Não obstante, conhecer os fatores associados aos diferentes tipos e intensidades de atividades físicas torna-se relevante para orientar programas de intervenção que visem promover o estilo de vida ativo durante a adolescência, especialmente aos grupos de maior risco ao comportamento sedentário.

Neste contexto, o presente estudo identificou o sexo, a faixa etária e a classe econômica como os principais fatores associados à prática de atividades físicas dos adolescentes investigados. Esta associação foi observada para o sexo na prática semanal de caminhada e de atividades físicas vigorosas, para a faixa etária na prática de caminhada, e para a classe econômica na prática de atividades físicas moderadas. Estes resultados evidenciaram a importância de se considerar os diferentes tipos e intensidades de atividades físicas para compreender os fatores associados com a prática de atividade física em adolescentes.

Os achados do presente estudo diferem do que tem sido descrito na literatura com relação aos fatores associados à prática de atividade física em adolescentes. É bem relatado na literatura que adolescentes do sexo feminino são menos ativas do que seus pares do sexo masculino (Butcher, Sallis, Mayer, \& Woodruff, 2008; Ceschini et al., 2009; Guedes, Guedes, Barbosa, \& Oliveira, 2001; Marani et al., 2006; Nader, Bradley, Houts, McRitchie, \& O'Brien, 2008; Oehlschlaeger, Pinheiro, Horta, Gelatti, \& San'Tana, 2004; Pahkala et al., 2008). Contudo, ao comparar os sexos no presente estudo, verificou-se que as meninas apresentaram maior tempo semanal do que os meninos na prática de caminhada e atividades físicas vigorosas, embora não tenha havido diferença significativa entre os sexos na proporção de adolescentes inativos, quando analisada a prática total de atividade física. Os resultados do presente estudo corroboram com outros que verificaram maior prática de atividade física em adolescentes do sexo feminino de uma cidade na Espanha (Casterad et al., 2006) e de uma comunidade rural em Moçambique (Nhantumbo et al., 2008). Além disso, estudo prévio realizado com adolescentes de dez cidades européias também identificou que meninas apresentavam maior tempo despendido na prática de caminhada em comparação aos rapazes (De Cocker et al., 2011).

Visando uma melhor compreensão destes achados, deve-se considerar que mais de $90 \%$ da amostra do presente estudo foi composta por adolescentes de classe econômica baixa ou média. Nesse contexto, estudos prévios indicam que a prática de atividades intensas de organização da casa (lavar roupa, limpeza da casa, dentre outras) é mais frequentemente realizada por jovens de classe econômica baixa, principalmente do sexo feminino (De Cocker et al., 2011; Farias Júnior, 2008; Gonçalves et al., 2007; Nhantumbo et al., 2008; Prista, Marques, \& Maia, 1997). Estas evidências podem explicar, em parte, os achados do presente estudo em relação às diferenças entre os sexos para a prática de atividades físicas. Contudo, considerando que grande parte dos estudos indica que adolescentes do sexo masculino são mais ativos do que seus pares do sexo feminino, a extrapolação destes resultados deve ser realizada com cautela. Nesse sentido, sugere-se a realização de outras investigações que comparem os diferentes tipos e intensidades de atividades físicas entre os sexos.

Quanto à relação entre atividade física e faixa etária, observou-se que os adolescentes mais velhos apresentaram maior tempo semanal na prática de caminhada do que os adolescentes das faixas etárias menores. Embora alguns estudos apontem uma maior prática de atividade física em adolescentes mais velhos (Nhantumbo et al., 2008; Vasconcelos \& Maia, 2001), preconiza-se na literatura que adolescentes mais novos são mais ativos (Bucther et al., 2008; Ceschini et al., 2009; Guedes et al., 2001; Oehlschlaeger et al., 2004). Contudo, vale ressaltar que a 
maioria dos estudos analisou a prática de atividade física total, enquanto o presente estudo identificou que adolescentes mais velhos apresentaram resultados significativamente superiores somente para a prática de caminhada. Nesse sentido, cabe mencionar que a prática de caminhada como meio de transporte é bastante comum em jovens de classe econômica baixa ou média (Bastos et al., 2008; Hallal et al., 2006; Nhantumbo et al., 2008), as quais são representadas pela maioria dos adolescentes do presente estudo. Além disso, os pais/responsáveis permitem que adolescentes mais velhos façam deslocamentos a pé sozinhos ou com amigos, enquanto os mais novos caminham menos por necessitarem do acompanhamento de adultos ao realizar esta atividade física, devido principalmente aos problemas de segurança pública (violência, assaltos, acidentes, etc.) (Gonçalves et al., 2007; Gordon-Larsen et al., 2000). Estes fatores possivelmente favoreceram o maior tempo despendido na prática de caminhada nos adolescentes mais velhos.

A classe econômica foi o único fator associado com a prática de atividades físicas moderadas, sendo que os adolescentes de classe econômica mais elevada apresentaram dispêndio de tempo semanal superior em atividades moderadas em relação aos seus pares de classes econômicas menos privilegiadas. Alguns estudos sugerem que jovens de classe econômica mais elevada participam com maior frequência de atividades físicas que geralmente apresentam intensidade moderada, como práticas esportivas organizadas (escolinhas de modalidades esportivas, clubes, etc.) e espontâneas (atividade física de lazer) (Hallal et al., 2006; Mota \& Sallis, 2002; Seabra et al., 2008), o que possivelmente contribuiu para as diferenças entre as classes econômicas observadas no presente estudo.

O estado nutricional e a escolaridade do pai não estiveram associados com a prática de caminhada e com atividades físicas moderadas e vigorosas na presente investigação. Por outro lado, estudos prévios observaram associação destes fatores com a prática de atividade física em adolescentes (Bastos et al., 2008; Butcher et al., 2008; Farias Júnior, 2008; GordonLarsen et al., 2000; Oehlschlaeger et al., 2004; Vasques \& Lopes, 2009). Entretanto, deve-se considerar que estes estudos consideraram a prática de atividade física total. Neste sentido, sugere-se a realização de outros estudos visando identificar a associação do estado nutricional e da escolaridade dos pais com diferentes tipos e intensidades de atividades físicas.

A principal contribuição do presente estudo foi a análise de fatores associados com os diferentes tipos e intensidades de atividade física (prática de caminhada, atividades físicas moderadas e vigorosas) na adolescência, tendo em vista que a maioria dos estudos presentes na literatura investigou a quantidade total de atividade física. Contudo, o presente estudo possui limitações que precisam ser consideradas. A primeira está relacionada à utilização do IPAQ em adolescentes menores de 14 anos de idade, uma vez que este questionário não apresenta boa reprodutibilidade e validade para adolescentes brasileiros desta faixa etária (Guedes et al., 2005). Além disso, o presente estudo foi realizado com adolescentes de apenas uma escola pública do município de Fortaleza, Ceará, não representando a população deste município. Portanto, deve-se ter cautela na generalização dos resultados.

\section{CONCLUSÕES}

Os resultados do presente estudo evidenciaram que os fatores associados com a prática de atividade física em adolescentes variam de acordo com o tipo e intensidade de atividade realizada. $O$ sexo apresentou-se associado com a prática de caminhada e de atividades físicas vigorosas (maior tempo de prática semanal para o sexo feminino), enquanto a faixa etária esteve associada somente com a prática de caminhada (maior tempo de prática semanal para os adolescentes 
mais velhos). Além disso, a classe econômica foi associada com a prática de atividades físicas moderadas (maior tempo de prática semanal para a classe mais elevada). Desta forma, podese sugerir que questões demográficas e econômicas podem influenciar a prática de atividades físicas de adolescentes. Os achados do presente estudo precisam ser confirmados em investigações futuras para direcionar a implantação de políticas públicas de promoção de atividade física para a população jovem.

\section{REFERÊNCIAS}

Associação Brasileira de Empresas de Pesquisa ABEP (2008). Critério de classificação econômica Brasil. São Paulo: Autor.

Bastos, J. P., Araújo, C. L., \& Hallal, P. C. (2008). Prevalence of insufficient physical activity and associated factors in Brazilian adolescents. Journal of Physical Activity and Health, 5(6), 777794.

Boreham, C., Twisk, J., Neville, C., Savage, M., Murray, L., \& Gallagher, A. (2002). Associations between physical fitness and activity patterns during adolescence and cardiovascular risk factors in young adulthood: The Northern Ireland Young Hearts Project. International Journal of Sports Medicine, 23(S1), S22-S26.

Butcher, K., Sallis, J. F., Mayer, J. A., \& Woodruff, S. (2008). Correlates of physical activity guideline compliance for adolescents in 100 U.S. cities. Journal of Adolescent Health, 42 (4), 360-368.

Caspersen, C. J., Powell, K. E., \& Christenson, G. M. (1985). Physical activity, exercise, and physical fitness: Definitions and distinctions for healthrelated research. Public Health Reports, $100(2)$, 126-131.

Casterad, J. A., Puyal, J. R., Gurrola, O. C., Lanaspa, E. G., Ostariz, E. S., \& Clemente, J. A. J. (2006). Los factores ambientales y su influencia en los patrones de actividad física en adolescentes. Revista Internacional de Ciencias del Deporte, 4(2), 1-14.

Ceschini, F. L., Andrade, D. R., Oliveira, L. C., Araújo Júnior, J. F., \& Matsudo, V. K. (2009). Prevalência de inatividade física e fatores associados em estudantes do ensino médio de escolas públicas estaduais. Jornal de Pediatria, 85(4), 301-306.
Conde, W. L., \& Monteiro, C. A. (2006). Body mass index cutoff points for evaluation of nutritional status in Brazilian children and adolescents. Jornal de Pediatria, 82 (4), 266-272.

De Cocker, K., Ottevaere, C., Sjostrom, M., Moreno, L., Warnberg, J., Valtueña, J., ... Bourdeaudhuij, I. (2011). Self-reported physical activity in European adolescents: Results from the HELENA (Health Lifestyle in Europe by Nutrition in Adolescence) study. Public Health Nutrition, 14(2), 246-254.

Farias Júnior, J. C. (2008). Associação entre prevalência de inatividade física e indicadores de condição socioeconômica em adolescentes. Revista Brasileira de Medicina do Esporte, 14(2), 109-114.

Gonçalves, H., Hallal P. C., Amorim, T. C., Araújo, C. L., \& Menezes, A. M. (2007). Fatores socioculturais e nível de atividade física no início da adolescência. Revista Panamericana de Salud Publica, 22 (4), 246-253.

Gordon, C. C., Chumlea, W. C., \& Roche, A. F. (1988). Stature, recumbent length, and weight. In T. G. Lohman, A. F. Roche, \& R. Martorell (Eds.), Anthropometric standardization reference manual (pp. 3-8). Champaign, IL: Human Kinetics.

Gordon-Larsen, P., McMurray, R. G., \& Popkin, B. M. (2000). Determinants of adolescent physical activity and inactivity patterns. Pediatrics, 105(6), 83-92.

Guedes, D. P., Guedes, J. E., Barbosa, D. S., \& Oliveira, J. A. (2001). Níveis de prática de atividade física habitual em adolescentes. Revista Brasileira de Medicina do Esporte, 7 (6), 187198.

Guedes, D. P., Lopes, C. C., \& Guedes, J. E. (2005). Reprodutibilidade e validade do Questionário Internacional de Atividade Física em adolescentes. Revista Brasileira de Medicina do Esporte, 11(2), 151-158.

Hallal, P. C., Bertoldi, A. D., Gonçalves, H., \& Victora, C. G. (2006). Prevalência de sedentarismo e fatores associados em adolescentes de 10-12 anos de idade. Cadernos de Saúde Pública, 22 (6), 1277-1287.

IPAQ Research Committee (2005). Guidelines for data processing and analysis of the International Physical Activity Questionnaire (IPAQ). Consultado em 20 de Agosto de 2008, a partir de http://www.ipaq.ki.se/ 
Katzmarzyk, P. T., Baur, L. A., Blair, S. N., Lambert, E. V., Oppert, J. M., \& Riddoch, C. (2008). International conference on physical activity and obesity in children: Summary statement and recommendations. Applied Physiology, Nutrition and Metabolism, 33 (2), 371-388.

Kvaavik, E., Klepp, K., Tell, G. S., Meyer, H. E., \& Batty, G. D. (2009). Physical fitness and physical activity at age 13 years as predictors of cardiovascular disease risk factors at ages 15, 25, 33, and 40 years: Extended follow-up of the Oslo Youth Study. Pediatrics, 123 (1), 80-86.

Marani, F., Oliveira, A. R., \& Guedes, D. P. (2006). Indicadores comportamentais associados à prática de atividade física e saúde em escolares do ensino médio. Revista Brasileira de Ciência e Movimento, 14(4), 63-70.

Matsudo, S. M., Araújo, T. L., Matsudo, V. K., Andrade, D., Andrade, E., Oliveira, L. C., \& Braggion, G. (2001). Questionário Internacional de Atividade Física (IPAQ): Estudo de validade e reprodutibilidade no Brasil. Revista Brasileira de Atividade Física \& Saúde, 6(2), 5-18.

Mota, J., \& Sallis, J. (2002). Actividade física e saúde: Factores de influência da actividade física nas crianças e nos adolescentes. Porto: Campo das Letras.

Murphy, M. H., McNeilly, A. M., \& Murtagh, E. M. (2010). Public health nutrition physical activity prescription for public health. Proceedings of the Nutrition Society, 69(1), 178-184.

Nader, P. R., Bradley, R. H., Houts, R. M., McRitchie, S. L., \& O’Brien, M. (2008). Moderate-to-vigorous physical activity from ages 9 to 15 years. The Journal of the American Medical Association, 300(3), 295-305.

Nhantumbo, L., Maia, J., Saranga, S., \& Prista, A. (2008). Atividade física em crianças e jovens residentes em uma comunidade rural moçambicana: Efeitos da idade, sexo e estado nutricional. Revista Panamericana de Salud Publica, 23(3), 171-178.

Oehlschlaeger, M. H., Pinheiro, R. T., Horta, B., Gelatti, C., \& San'Tana, C. (2004). Prevalência e fatores associados ao sedentarismo em adolescentes de área urbana. Revista de Saúde Pública, 38(2), 157-163.

Pahkala, K., Heinonen, O. J., Lagstrom, H., Hakala, P., Simell, O., \& Viikari, J. S. (2008). Vascular endothelial function and leisure-time physical activity in adolescents. Circulation, 118(23), 2353-2359.

Prista, A., Marques, A. T., \& Maia, J. (1997). Relationship between physical activity, socioeconomic status, and physical fitness of 815-year-old youth from Mozambique. American Journal of Human Biology, 9(4), 449-457.

Sallis, J. F., Prochaska, J. J., \& Taylor, W. C. (2000). A review of correlates of physical activity of children and adolescents. Medicine $\mathcal{E}$ Science in Sports \& Exercise, 32(5), 963-975.

Seabra, A. F., Mendonça, D. M., Thomis, M. A., Anjos, L. A., \& Maia, J. A. (2008). Determinantes biológicos e sócio-culturais associados à prática de atividade física de adolescentes. Cadernos de Saúde Pública, 24(4), 721-736.

Strong, W. B., Malina, R. M., Blimkie, C. J., Daniels, S. R., Dishman, R. K., Gutin, B., ... Trudeau, F. (2005). Evidence based physical activity for school-age youth. The Journal of Pediatrics, 146(6), 732-737.

Twisk, J. W., Kemper, H. C., Van Mechelen, W., \& Post, G. B. (2001). Clustering of risk factors for coronary heart disease: The longitudinal relationship with lifestyle. Annals of Epidemiology, 11(3), 157-165.

Vasconcelos, M. A., \& Maia, J. (2001). Actividade física de crianças e jovens - Haverá um declínio? Estudo transversal em indivíduos dos dois sexos dos 10 aos 19 anos de idade. Revista Portuguesa de Ciências do Desporto, 1 (3), 44-52.

Vasques, D. G., \& Lopes, A. S. (2009). Fatores associados à atividade física e aos comportamentos sedentários em adolescentes. Revista Brasileira de Cineantropometria $e$ Desempenho Humano, 11 (1), 59-66.

(c) EY-No Todo o conteúdo da revista Motricidade está licenciado sob a Creative Commons, excepto quando especificado em contrário e nos conteúdos retirados de outras fontes bibliográficas. 